\title{
Estratégia lúdica no processo de ensino e aprendizagem de Ciências em escola
}

\section{pública de Santarém-PA}

\author{
Playful strategy in the Science teaching and learning process in a public school in Santarém-PA \\ Estrategia lúdica en el proceso de enseñanza y aprendizaje de las Ciencias en una escuela pública \\ de Santarém-PA
}

Maria Vicência Penaforte Maia

ORCID: https://orcid.org/0000-0003-1022-5679

Centro Universitário da Amazônia, Brasil E-mail: maria2016maia@gmail.com

Milena Dias Dorabiato

ORCID: https://orcid.org/0000-0003-1972-7739

Universidade Federal do Oeste do Pará, Brasil

E-mail: milenadorabiato@hotmail.com

Susane Marinho Lages

ORCID: https://orcid.org/0000-0002-2877-0678

Universidade Federal do Oeste do Pará, Brasil E-mail: susanelages@hotmail.com

\section{Resumo}

Estratégias lúdicas contribuem para uma aprendizagem dinâmica e significativa por despertar o interesse e a atenção dos alunos de forma descontraída. Diante disso, o presente trabalho objetivou identificar as contribuições do jogo didático "sangue que salva", associado à aula expositiva, para o ensino de Ciências a educandos do $8^{\circ}$ ano do ensino fundamental de escola pública em Santarém-PA. O tema escolhido, de acordo com o plano de ensino, foi "Tipos 
sanguíneos, fator Rh e sistema imunológico”. Antes das atividades teórica e lúdica, foi aplicado um pré-teste para sondar o conhecimento prévio dos alunos sobre os temas e, logo após, ocorreu a aplicação da aula teórica. Em seguida, o jogo foi aplicado para 23 alunos de duas turmas (A e B) do $8^{\circ}$ Ano do ensino fundamental. Após o jogo didático, foi aplicado um pós-teste a fim de verificar a contribuição da atividade lúdica, associada a aula teórica, no processo de aprendizagem. O jogo foi realizado em duplas, cada dupla recebeu uma cartela que representava uma bolsa de sangue cheia. Conforme a metodologia e regras do jogo, as duplas deveriam avaliar a ocorrência de possibilidade de transfusões sanguíneas. Os resultados obtidos pela análise dos testes antes e depois das atividades teórica e lúdica foram satisfatórios. Houve um aumento percentual nas médias de notas do pós-teste em relação ao pré-teste em ambas as turmas, na turma A o aumento foi de $35 \%$ e na turma B de 42,65\%. A estratégia lúdica "Sangue que salva" proporcionou um espaço de interação e aprendizado aos alunos, possibilitando a construção do conhecimento e contribuindo, assim, para o processo de ensino e aprendizagem, assim como para o desenvolvimento de habilidades, além de tornar o processo educacional mais interessante e divertido.

Palavras-chave: Conhecimento prévio; Construção do conhecimento; Ensino; Jogos; Metodologia de ensino.

\begin{abstract}
Playful strategies contribute to dynamic and meaningful learning by arousing students' interest and attention in a relaxed way. Therefore, this study aimed to identify the contributions of the didactic game "blood that saves", associated with the expositive class, for the teaching of Science to 8th grade students of public school in SantarémPA. The chosen topic, according to the teaching plan, was "Blood types, Rh factor and immune system". Before the theoretical and recreational activities, a pre-test was applied to probe the students' previous knowledge about the themes and, soon after, the theoretical class was applied. Then, the game was applied to 23 students from two classes (A and B) of the 8th year of elementary school. After the didactic game, a post-test was applied in order to verify the contribution of the playful activity, associated with the theoretical class, in the learning process. The game was played in pairs, each pair received a card that represented a full blood bag. According to the methodology and rules of the game, the pairs should assess the occurrence of the possibility of blood transfusions. The results obtained by analyzing the tests before and after the theoretical and recreational activities were satisfactory. There was a percentage increase in the average grades of the post-test in relation to the pre-test in both classes, in class A the increase was $35 \%$ and in class B, $42.65 \%$. The playful strategy "blood that saves" provided a space for interaction and learning for students, enabling the construction of knowledge and thus contributing to the teaching and learning process, as well as to the development of skills, in addition to making the educational process more interesting and fun.
\end{abstract}

Keywords: Previous knowledge; Construction of knowledge; Teaching; Games; Teaching methodology.

\title{
Resumen
}

Las estrategias lúdicas contribuyen al aprendizaje dinámico y significativo al despertar el interés y la atención de los estudiantes de una manera relajada. Por tanto, este estudio tuvo como objetivo identificar los aportes del juego didáctico "sangue que salva", asociado a la clase expositiva, para la enseñanza de la ciencia a alumnos de $8^{\circ}$ grado de la escuela pública de Santarém-PA. El tema elegido, según el plan docente, fue "Tipos de sangre, factor Rh y sistema inmunológico". Previo a las actividades teóricas y recreativas, se aplicó un pre-test para sondear los conocimientos previos de los estudiantes sobre los temas y, poco después, se aplicó la clase teórica. Luego, el juego se aplicó a 23 alumnos de dos clases (A y B) del $8^{\circ}$ año de primaria. Tras el juego didáctico, se aplicó un post-test para comprobar el aporte de la actividad lúdica, asociada a la clase teórica, en el proceso de aprendizaje. El juego se jugó en parejas, cada pareja recibió una tarjeta que representaba una bolsa de sangre llena. De acuerdo con la metodología y las reglas del juego, las parejas deben evaluar la ocurrencia de la posibilidad de transfusiones de sangre. Los resultados obtenidos al analizar las pruebas antes y después de las actividades teóricas y recreativas fueron satisfactorios. Hubo un aumento porcentual en las calificaciones promedio del post-test en relación al pre-test en ambas clases, en la clase A el incremento fue del $35 \%$ y en la clase B, del 42,65\%. La estrategia lúdica "Sangue que salva" brindó un espacio de interacción y aprendizaje para los estudiantes, posibilitando la construcción del conocimiento y contribuyendo así al proceso de enseñanza y aprendizaje, así como al desarrollo de habilidades, además de hacer más el proceso educativo. interesante y divertido.

Palabras clave: Conocimiento previo; Construcción de conocimiento; Enseñanza; Juegos; Metodología de la enseñanza.

\section{Introduçãa}

O ensino de ciências, bem como o da biologia, enfrenta muitos desafios no cenário atual, sendo uma das áreas de estudo em que os alunos apresentam maior grau de dificuldade quanto à assimilação e compreensão de conceitos destas disciplinas. Este cenário leva ao desinteresse e a falta de motivação dos alunos para o estudo das ciências biológicas. Paim, Goldschmidt e Loreto (2021, p.2) afirmam sobre algumas questões relacionadas a algumas destas dificuldades: 
Questões como: a dificuldade de abstração dos conteúdos pelos jovens; a tendência predominante em um ensino memorístico, focado em decorar e não construir aprendizado; a presença de nomes complexos e totalmente novos para os alunos; a falta em estabelecer uma ligação entre o conhecimento ensinado e o cotidiano dos alunos; e, a necessidade em despertar o interesse dos alunos com aulas mais atrativas.

A disciplina, portanto, vem apresentando um caráter de memorização de nomes, estruturas, funções e processos celulares sem que o conhecimento acerca desses assuntos seja construído e relacionado à vida cotidiana de nossos alunos. Esses fatores podem influenciar na diminuição do nível de interesse dos alunos do ensino fundamental pela disciplina de Ciências. Marques (2018, p.1) fala sobre isso:

O Ensino de Ciências é marcado por inúmeras abstrações e memorização de nomenclaturas, esses são alguns dos motivos que repelem parte dos estudantes de gostar de disciplinas como Biologia. Diante desta problemática, é necessário investir em alternativas didáticas que visem a atrair os discentes e a facilitar os processos de aprendizagem perante os conteúdos abstratos.

Segundo Paim et al. (2021), a área da biologia na qual os alunos mais apresentam dificuldades é a biologia celular. Isso se deve ao fato da grande quantidade de conceitos, de difícil visualização, e que, muitas vezes, o conhecimento prévio relacionado ao assunto está ausente ou é insuficiente. Observa-se isso no estudo das células nas aulas de ciências que, ainda hoje, com tantas inovações metodológicas, continuam sendo trabalhadas, em muitas escolas, de forma abstrata, utilizando-se de métodos de ensino pouco atrativos e que não despertam o interesse e a atenção, desmotivando e distanciando cada vez mais os alunos do estudo desta disciplina.

Desta forma, faz-se necessário o uso de recursos educacionais que facilitem a visualização e a compreensão dos conteúdos abstratos, bem como que façam a contextualização desses assuntos ao cotidiano dos alunos. Diante deste cenário, é essencial a aplicação de metodologias de ensino que possam auxiliar o trabalho do professor, visando motivar o aluno e contribuir para uma aprendizagem significativa (Pereira, Azevedo, Sousa, Hager, 2020).

Freitas Filhos, Freitas, Silva e Melo (2012) asseguram que o objetivo da atividade lúdica não é apenas levar o estudante a memorizar mais facilmente o assunto abordado, mas sim induzir o raciocínio do aluno, a reflexão, o pensamento e consequentemente a construção do seu conhecimento, onde promove a construção do conhecimento cognitivo, físico, social e psicomotor. Assim, o desenvolvimento de metodologias de ensino que faça uso da ludicidade pode auxiliar nesse processo, sendo um importante recurso metodológico a ser utilizado em sala de aula, neste caso em especial, no ensino de ciências, possibilitando a interação do aluno com o objeto de estudo, em um processo criativo e construtivo do conhecimento, estimulando um espaço coletivo de integração, socialização, participação e engajamento dos alunos durante as aulas, possibilitando, desta forma, o desenvolvimento do processo de ensino-aprendizagem.

A aplicação das atividades do Projeto "Atividades Lúdicas: uma abordagem diferenciada para o processo de ensinoaprendizagem em Biologia Celular" nas aulas de Ciências para o $8^{\circ}$ ano da Escola Municipal de Ensino Fundamental Paulo Rodrigues dos Santos, no município de Santarém-PA, teve o objetivo de desenvolver e aplicar estratégias lúdicas para estabelecer uma investigação a respeito do uso da ludicidade para o ensino de ciências, nesta pesquisa abordando o assunto "Sistema imunológico, Fator Rh, Tipagem sanguínea", procurando identificar as contribuições das atividades lúdicas como estratégias para o processo de ensino e aprendizagem em ciências, bem como para despertar o interesse e a motivação dos alunos para o estudo dos conteúdos das ciências biológicas. 


\section{Metodologia}

O presente trabalho trata-se de um estudo exploratório com abordagem quali-quantitativa (Gil, 2008). Estudos exploratórios englobam procedimentos como revisões bibliográficas e análise inicial de variáveis qualitativa e/ou quantitativa, com objetivo de proporcionar maior familiaridade com os objetos de investigação (Gil, 2008). No particular estudo, investigase possíveis efeitos que um conjunto de atividades lúdicas podem ter sobre a aprendizagem dos alunos - requerendo variáveis qualitativa (engajamento dos alunos durante as atividades) e quantitativa (desempenho em um teste avaliativo antes e após implementação das atividades lúdicas).

A atividade foi realizada, na disciplina de Ciências, com 29 alunos do $8^{\circ}$ ano regular do Ensino Fundamental, da E.M.E.F. Paulo Rodrigues dos Santos, no município de Santarém, PA.

Após a explicação do projeto (Figura 1A e 1B), foi apresentado aos alunos o TCLE (Termo de Consentimento livre e Esclarecido), a fim de informa-los a sua participação na pesquisa, termo este, que foi encaminhado aos pais para que autorizassem, tendo eles a liberdade de aceitar ou não participar do trabalho.

Figura 1A e 1B: Apresentação do projeto às turmas $8^{\circ}$ ano A e B da Escola Municipal Paulo Rodrigues.
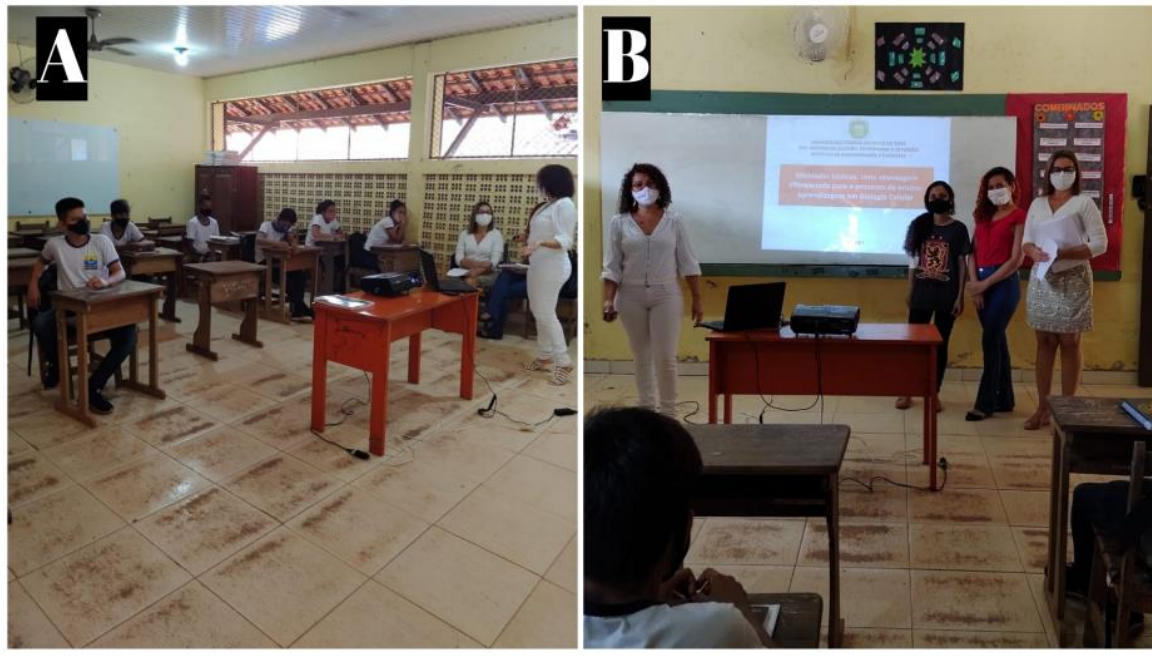

Fonte: Autores.

A aplicação do projeto foi dividida em cinco etapas: aplicação de pré-teste; aulas teóricas (Figura 2A e 2B); estratégia lúdica; aplicação de pós-teste e, aplicação do questionário de avaliação de metodologia. Em cada turma, foram ministradas 4 aulas, 2 por semana, com a duração de 40 minutos cada, de acordo com o Quadro 1.

A escolha das turmas foi realizada juntamente com a professora responsável pela disciplina e o conteúdo escolhido, de acordo com o plano de ensino: tipos sanguíneos, fator Rh e sistema imunológico. Participaram da pesquisa 29 alunos de duas turmas do $8^{\circ}$ ano, sendo: 14 alunos da turma A, 15 alunos da turma B. Em ambas as turmas, foram ministradas aulas teóricas/estratégia lúdica, embasado em livro-texto utilizado na educação básica e, pesquisa em periódicos. Inicialmente, o conteúdo foi ministrado por meio de aula expositiva, com o auxílio de projetor de imagem, utilização de vídeo e animações. 
Figura 2A e 2B: Apresentação do projeto nas turmas $8^{\circ}$ A e B da Escola Municipal Paulo Rodrigues.

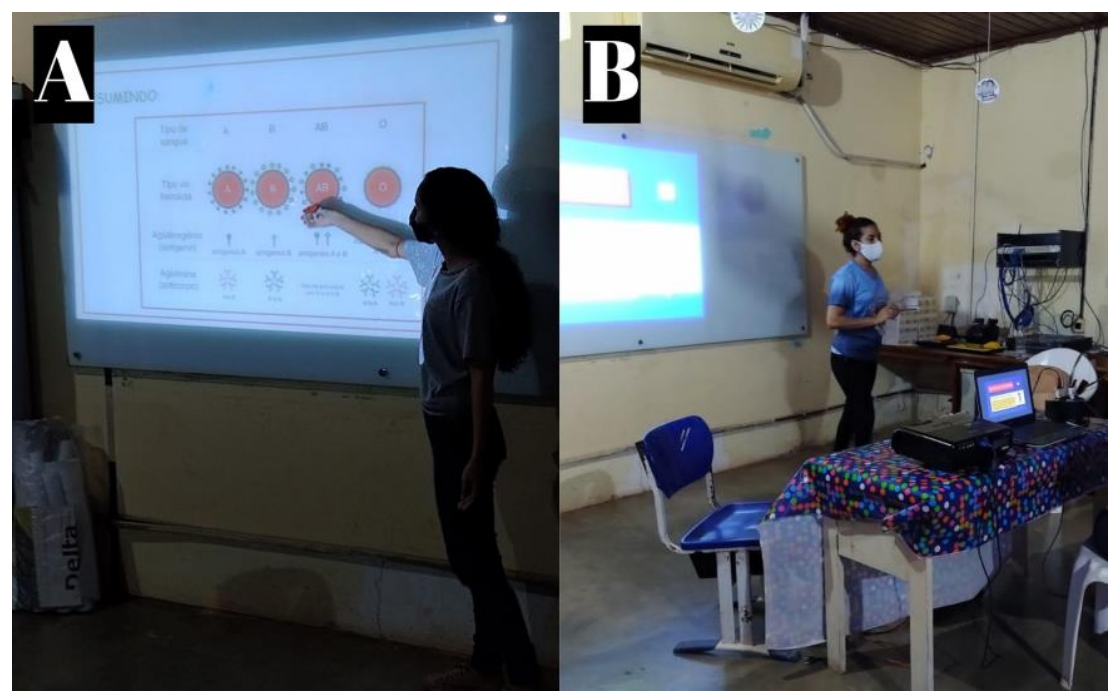

Fonte: Autores.

Quadro 1: Cronograma de aplicação do projeto nas turmas A e B.

\begin{tabular}{|ccccc|}
\hline SÉRIE & SALA & TURMA & 3 $^{\text {a }}$ FEIRA & Data da aplicação \\
\hline \multirow{2}{*}{$8^{\circ}$ ANO } & 801 & A & $14: 50-16: 10$ & $14 / 09$ e $21 / 09$ \\
& 802 & B & $16: 10-17: 15$ & $14 / 09$ e $21 / 09$ \\
\hline
\end{tabular}

Fonte: Autores.

Após a aula teórica, foi realizada a estratégia lúdica para a qual, foi confeccionado um jogo "sangue que salva" (Figura 3A), de acordo com Pliessnig (2013).

\section{Peças do Jogo "Sangue que salva"}

O jogo foi composto por:

1 - Um cartão com o desenho de uma "bolsa de sangue" e uma cartela interna que deslizava para fora e para dentro, que representavam a bolsa cheia ou vazia de sangue (Figura 3B);

2- Três dados que representavam a "presença" ou "ausência de reação" entre o sangue e os soros: Anti A, Anti-B e Anti-Rh. Os três juntos determinavam o "sangue testado" (Figura 3C);

3 - Um dado que indicava o "sentido da doação", contendo 4 diferentes possibilidades (Figura 3C);

4 - Um dado octaédrico, contendo os 8 tipos sanguíneos do Sistema ABO e Fator Rh, que determinava o "sangue conhecido" (Figura 3C). 
Figura 3A, 3B e 3C: Confecção e montagem das peças do jogo.

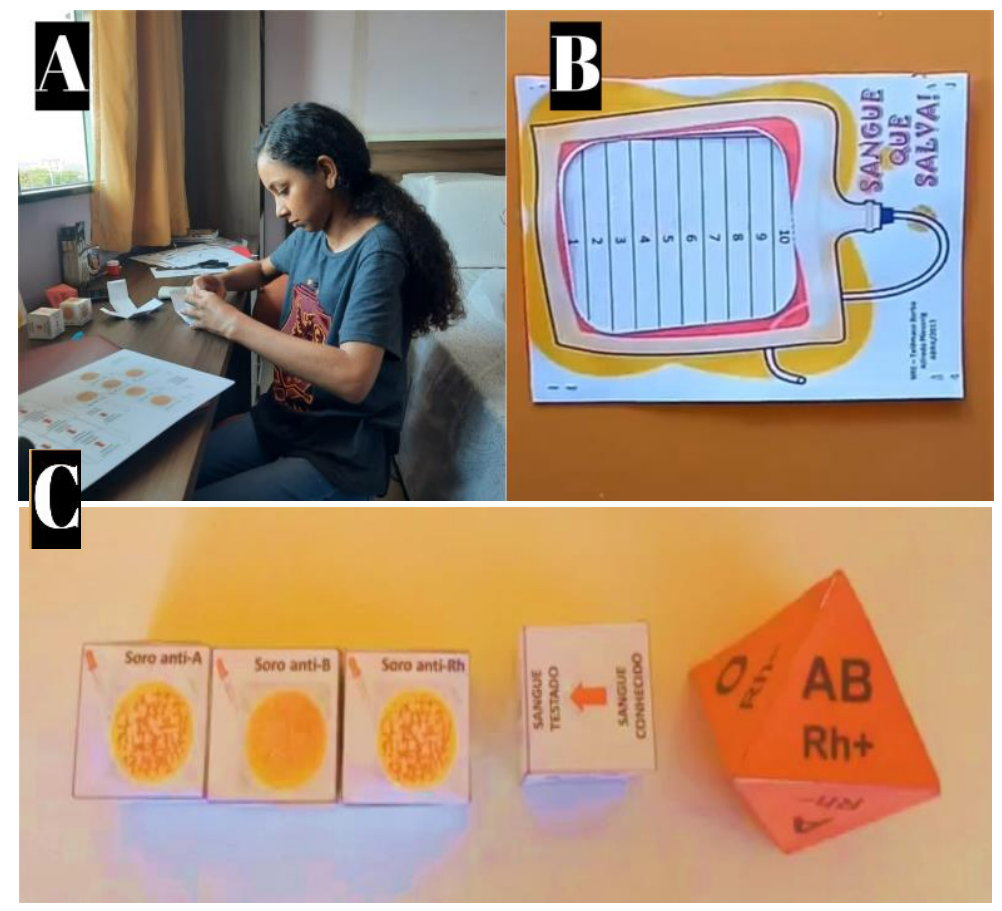

Fonte: Autores.

O jogo foi realizado em duplas, dispostas em semicírculo (Figura 4A e 4B). Cada dupla recebeu um kit contendo um cartão com a bolsa de sangue, uma cartela interna e cinco dados.

Figura 4A e 4B: Aplicação da estratégia lúdica nas turmas $8^{\circ} \mathrm{A}$ e $8^{\circ} \mathrm{B}$.

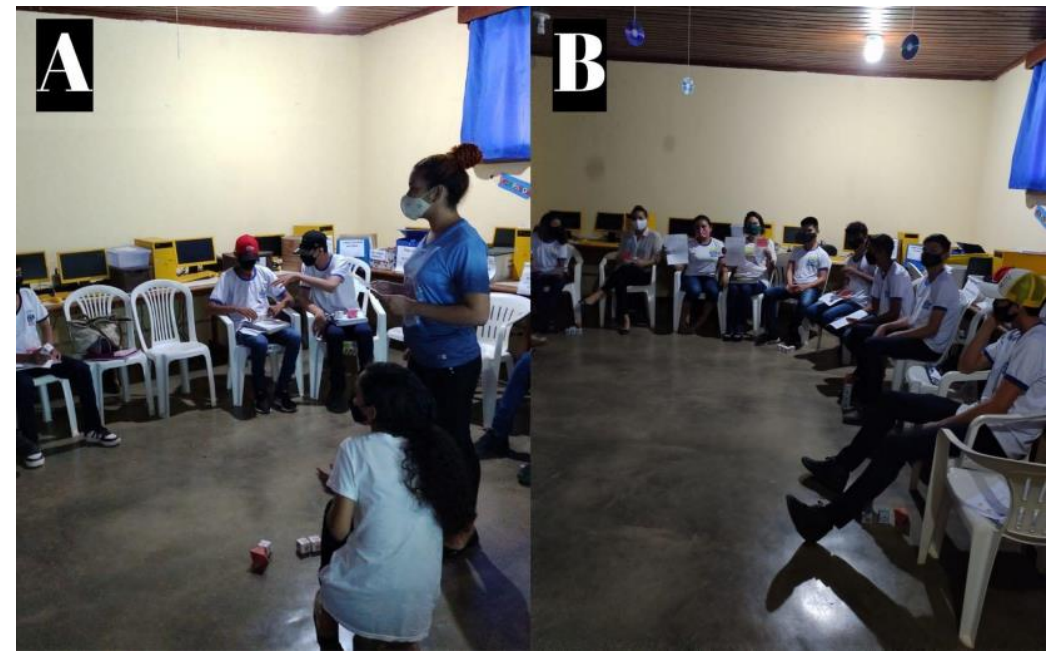

Fonte: Autores.

No início do jogo, cada dupla deveria retirar a cartela interna para representar a "bolsa cheia de sangue" (Figura 5A). Iniciava-se a partida com a dupla que primeiro obtivesse o tipo sanguíneo " $\mathrm{O} \mathrm{Rh}$, doador universal" ao jogar o dado octaédrico (sangue conhecido). A próxima dupla a jogar seria a dupla posterior à inicial, no sentido anti-horário, e assim por diante. Cada dupla, na sua vez, deveria lançar os cinco dados simultaneamente. A seguir, analisar os dados 1 (soro Anti-A), 2 (soro Anti-B) 
e 3 (soro Anti-Rh) para determinar o tipo sanguíneo do "sangue testado", verificando a ocorrência ou não de reação dos soros, conforme o Quadro 2.

Quadro 2: Reações dos soros Anti-A, Anti-B e Anti-Rh e tipo sanguíneo testado.

\begin{tabular}{|c|c|c|c|}
\hline Dado 1 & Dado 2 & Dado 3 & \\
\hline $\begin{array}{c}\text { Soro } \\
\text { Anti-A }\end{array}$ & $\begin{array}{c}\text { Soro } \\
\text { Anti-B }\end{array}$ & $\begin{array}{c}\text { Soro } \\
\text { Anti-Rh }\end{array}$ & Tipo Sanguíneo \\
\hline sem reação & sem reação & sem reaçăo & O Rh - \\
\hline sem reação & sem reação & com reação & $\mathrm{ORh}+$ \\
\hline com reação & sem reação & sem reação & A Rh - \\
\hline com reação & sem reação & com reaçăo & $\mathrm{ARh}+$ \\
\hline sem reação & com reação & sem reação & B Rh - \\
\hline sem reação & com reação & com reação & $\mathrm{B} \mathbf{R h}+$ \\
\hline com reação & com reação & sem reação & AB Rh - \\
\hline com reação & com reação & com reação & $\mathrm{AB} \mathrm{Rh}+$ \\
\hline
\end{tabular}

Fonte: Pliessnig (2013).

Após determinar o sangue testado, a dupla deveria verificar o "sangue conhecido" no dado octaédrico e o "sentido da doação", no dado específico. Feito isso, a dupla deveria realizar uma análise comparativa (doador/receptor) e informar se a doação "poderia” ou "não” ocorrer no sentido indicado pelo dado "sentido da doação", com base no Quadro 3.

Quadro 3: Doação de sangue, quem pode doar e quem pode receber cada tipo sanguíneo.

\begin{tabular}{|c|c|c|c|c|c|c|c|c|c|}
\hline & \multicolumn{8}{|c|}{ DOADOR } \\
\hline & & o- & $0+$ & A- & At & B- & $\mathrm{B}+$ & $A B-$ & $\mathrm{AB}+$ \\
\hline \multirow{8}{*}{ 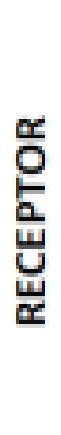 } & $0-$ & $x$ & & & & & & & \\
\hline & $0+$ & $\mathbf{x}$ & $x$ & & & & & & \\
\hline & $A=$ & $x$ & & $x$ & & & & & \\
\hline & $\mathrm{A}+$ & $x$ & $x$ & $x$ & $x$ & & & & \\
\hline & B- & $x$ & & & & $x$ & & & \\
\hline & $B+$ & $x$ & $x$ & & & $x$ & $x$ & & \\
\hline & $A B=$ & $x$ & & $x$ & & $x$ & & $x$ & \\
\hline & $A B+$ & $x$ & $x$ & $x$ & $x$ & $x$ & $x$ & $x$ & $x$ \\
\hline
\end{tabular}

Fonte: Pliessnig (2013).

Quando a doação era possível e, a dupla identificava corretamente, empurrava a aba da cartela na bolsa de sangue até o nível 5, indicando que essa parte do sangue havia sido doada (Figura 5B). Em seguida, passava a vez para próxima dupla. Caso a dupla da vez não percebesse a possibilidade de doação e outra dupla sim, aquela que primeiro indicasse a possibilidades de doação, poderia diminuir 5 níveis na sua bolsa de sangue. Nos casos em que a doação não era possível, embora a dupla respondesse corretamente, a cartela não era empurrada na bolsa de sangue e, a vez era passada para a próxima dupla. Ao final, a dupla que conseguisse esvaziar primeiro a bolsa de sangue (Figura 5C), vencia o jogo. 
Ao finalizar estas etapas, na aula seguinte, foi aplicado o pós-teste com objetivo de avaliar o conhecimento dos alunos referente aos conteúdos ministrados na ação extensionista aqui relatada. Ao final, os alunos também responderam a um questionário de avaliação da metodologia.

Figura 5A, 5B e 5C: Bolsa de sangue cheia (A), no nível 5 (B) e vazia (C).

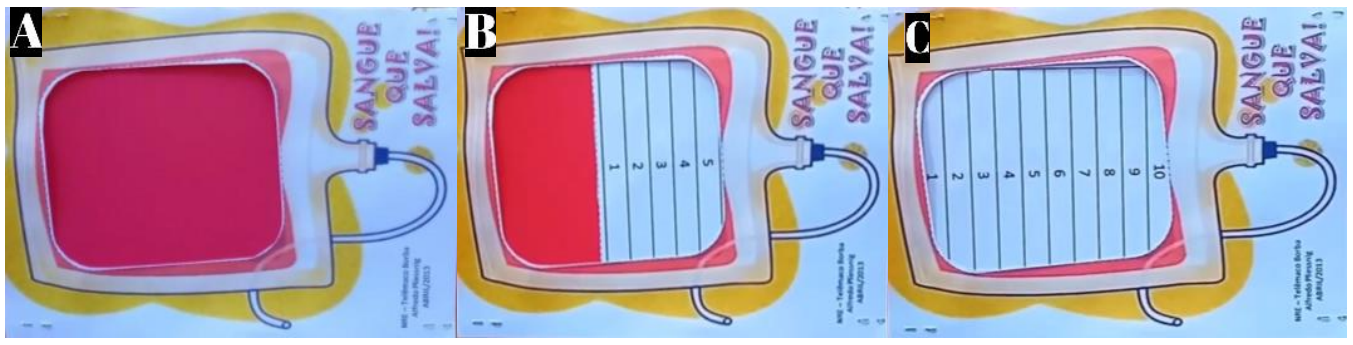

Fonte: Adaptada de Pliessnig (2013).

A análise do desempenho dos alunos no pré e pós-teste, a fim de avaliar efeitos da atividade lúdica sobre a aprendizagem dos alunos a respeito dos conteúdos abordados, foi realizada mediante tabulação das respostas dos alunos aos testes em uma planilha eletrônica. De posse dos dados tabulados, as respostas foram dicotomizadas, atribuindo-se "1" para respostas corretas e "0" para respostas incorretas, e analisadas por meio de estatística descritiva. Analisou-se precisão e poder discriminativo dos dois testes, com auxílio do coeficiente alfa de Cronbach e da medida de correlação ponto Bisserial. Quanto à análise das questões do teste, recorreu-se a proporção de acertos e erros, utilizado como indicativo de dificuldade na aprendizagem de conceitos mais específicos cobrados na questão.

Interpretação quanto ao nível de dificuldade do item de acordo com Condé (2001):

- $\quad$ item fácil: Acertos $>70 \%$;

- $\quad$ item de média dificuldade: $30 \%<$ acertos $\leq 70 \%$;

- $\quad$ item difícil: acertos $\leq 30 \%$.

Para comparação do desempenho médio dos alunos no pré e pós-teste recorreu-se à ANOVA (Análise de Variância) e ao teste d de Cohen (Espírito Santo \& Daniel, 2017).

\section{Resultados e Discussão}

O critério de inclusão dos sujeitos da amostra para a análise comparativa do desempenho médio observado antes e após a ação extensionista foi: ter realizado os dois testes. Assim, seis dos estudantes que realizaram apenas um dos testes foram excluídos da análise, o que resultou em uma amostra de $n=23$ estudantes.

A distribuição dos escores dos estudantes por turma, tanto no pré-teste quanto no pós-teste, podem ser visualizados no box plot apresentado na Figura 6. Observou-se que as medianas aumentaram em ambas as turmas, após a consecução das atividades lúdicas - indicativo de que o desempenho médio dos estudantes também aumentou no pós-teste.

$\mathrm{Na}$ turma $8^{\circ} \mathrm{A}$, observou-se que $50 \%$ das notas do pré-teste situaram-se entre 3 e 5 , com uma mediana de 4 e amplitude variando de 1 a 6. Já no pós-teste, 50\% das notas situaram-se entre 3,2 e 7, apresentando uma mediana de 5,5 e amplitude de 2 a 7,2.

Na turma $8^{\circ}$ B, $50 \%$ das notas no pré-teste situaram-se entre 5 e 6 , com uma mediana de 5 e amplitude variando de 4 a 7,2. Já no pós-teste, $50 \%$ das notas situaram-se entre 6 e 9, apresentando uma mediana de 8 e amplitude de 5 a 10. 
Esta avaliação permitiu inferir, para as duas turmas (A e B), que os resultados do pós-teste foram superiores ao préteste, com base nos valores da mediana, amplitude e concentração das notas. Semelhante ao observado por Duarte, Dorabiato, Azevedo e Hager (2020) que, ao avaliarem as contribuições de jogo lúdico no processo de ensino-aprendizagem, obtiveram os melhores valores no pós-teste em relação ao pré-teste. No entanto, Torres e Mendes (2019), verificaram que entre as medidas de pré-teste e pós-teste, para as três turmas avaliadas, não houve mudanças estatisticamente significantes no desempenho dos alunos.

Figura 6: Distribuição dos escores por turma: pré-teste e pós-teste

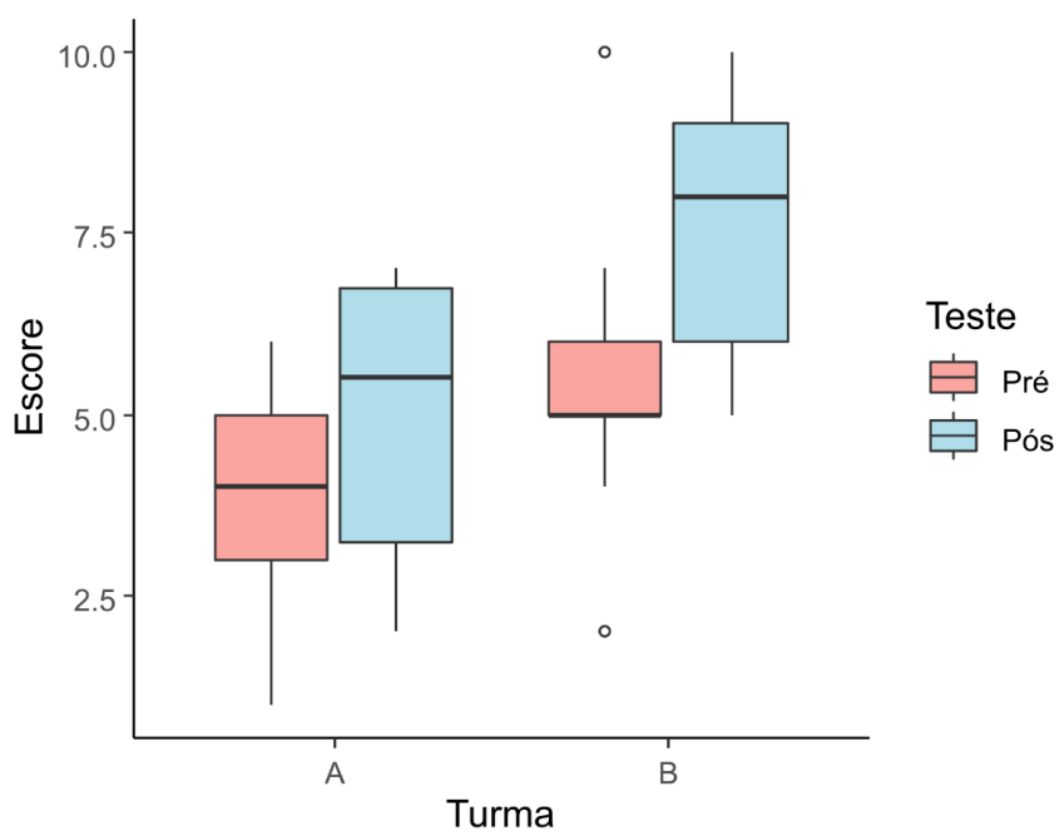

Fonte: Autores.

Observou-se que o desempenho dos alunos da turma $8^{\circ} \mathrm{B}$ foi maior que os da turma $8^{\circ} \mathrm{A}$. Resultado diferente ao observado por Torres e Mendes (2019) que ao comparar o desempenho entre três turmas observaram que não houve diferenças significativas entre elas, ou seja, que as turmas se mostraram equivalentes.

Observando o desempenho médio nos testes e o seu respectivo desvio padrão (Tabela 1), percebe-se que os alunos da turma $8^{\circ} \mathrm{B}$ obteve média mais elevada no pré-teste que o $8^{\circ} \mathrm{A}$. De acordo com Feijó e Delizoicov (2016), a diferença entre o conhecimento prévio dos alunos está relacionada, em parte, ao convívio social com pessoas de maior nível de escolaridade e, aos acessos aos meios de informação e comunicação.

Em relação ao pós-teste, verificou-se também maiores médias no pós-teste para os alunos da turma B em relação aos da turma A. 
Research, Society and Development, v. 11, n. 1, e13011124364, 2022

(CC BY 4.0) | ISSN 2525-3409 | DOI: http://dx.doi.org/10.33448/rsd-v11i1.24364

Tabela 1: Média e desvio padrão dos escores geral e por turma $(n=23)$

\begin{tabular}{c|cc|cc}
\hline \multirow{2}{*}{ Turma } & \multicolumn{2}{|c|}{ Pré-teste } & \multicolumn{2}{c}{ Pós-teste } \\
\cline { 2 - 5 } & Média & Desvio Padrão & Média & Desvio Padrão \\
\hline A & 4,000 & 1,886 & 5,400 & 2,757 \\
B & 5,231 & 2,006 & 7,462 & 1,613 \\
Geral & $\mathbf{4 , 6 9 6}$ & 2,010 & $\mathbf{6 , 5 6 5}$ & 2,371 \\
\hline
\end{tabular}

Fonte: Autores.

Comparando as médias de notas das turmas A e B, obtidas no pré-teste e pós-teste, observou-se que houve um aumento percentual de $35 \%$ e $42,65 \%$ na média do pós-teste, respectivamente. Resultados superiores aos obtidos por Ribeiro (2019), que obteve no pré-teste $(6,25)$; e no pós-teste $(7,54)$ do grupo experimental, aumento percentual de aproximadamente 20,64\% na média do pós-teste.

Desta forma, a média geral no pós-teste foi maior que a observada no pré-teste em ambas as turmas. Corroborando com Fenner e Corbari (2005) e Sousa et al. (2021) ao sugerirem que o conhecimento prévio do aluno deve servir de alicerce para a incorporação de novos conhecimentos. Assim, coube avaliar se as diferenças de desempenho das turmas eram estatisticamente significativas.

De posse dos escores, procedeu-se com a comparação do desempenho médio alcançado pelos estudantes que realizaram o pré-teste e o pós-teste. Para tanto, recorreu-se à Análise de Variância (ANOVA) de dois fatores (turma e teste). No entanto, uma comparação de médias por meio da ANOVA requer que alguns pressupostos sejam atendidos, a saber: i) normalidade das observações; ii) homogeneidade (Espírito Santo \& Daniel, 2017). Para verificar se os escores apresentados pelos estudantes seguem uma distribuição normal de probabilidade, realizou-se o teste de Shapiro-Wilk para um nível de significância $\alpha=0,05$, sob hipótese (H0) de que as observações (escores) estão normalmente distribuídas (Sousa, 2017). O resultado do teste, exibido na Tabela 2, mostra que os escores, observado por turma tanto no pré-teste quanto no pós-teste, seguem uma distribuição normal de probabilidade, uma vez que p.valor $>\alpha$.

Tabela 2: Teste de normalidade das observações $(\alpha=0,05)$

\begin{tabular}{ccc}
\hline Turma & Teste & p.valor \\
\hline A & Pré & 0,095 \\
B & Pré & 0,212 \\
A & Pós & 0,092 \\
B & Pós & 0,121 \\
\hline
\end{tabular}

Fonte: Autores.

No que se refere à homogeneidade, o teste de Bartlett retornou (K-squared =0,237, $\mathrm{df}=3$, p.valor $=0,971)$, indicando que o pressuposto da homogeneidade foi atendido (Sousa, 2017). Validados os pressupostos, partiu-se para a realização da ANOVA de dois fatores, visando identificar se havia diferença nos desempenhos apresentados por turmas (A e B) e em diferentes momentos da aplicação do teste (pré/pós).

A Tabela 3 exibe o resultado da ANOVA mencionada, na qual é expressa dois p-valor. O primeiro é resultado do teste ao fator Turma sobre o desempenho dos estudantes, isto é, se existe alguma diferença no desempenho dos estudantes do $8^{\circ}$ A 
em relação aos estudantes do $8^{\circ} \mathrm{B}$. Nota-se que o p-valor desse fator é muito pequeno $(0,009<0,05)$, levando à rejeição da hipótese nula, o que leva à constatação de diferença significativa no desempenho apresentado pelas diferentes turmas.

O segundo p-valor refere-se ao efeito que o fator "Teste" realizado antes (pré-teste) e posterior à atividade lúdica (pós-teste) exerceu no desempenho dos estudantes. Nesse fator também foi constatado p-valor também muito pequeno $(0,001<0,05)$, o que leva à rejeição da hipótese nula $\left(\mathrm{H}_{0}\right.$ : Não há influência do fator no desempenho dos sujeitos). Assim, assume-se a hipótese alternativa $\mathrm{H} 1$ ( $\mathrm{H}_{1}$ : há influência do fator no desempenho dos sujeitos) que postula diferenças significativas no desempenho apresentado pelos estudantes após participarem da atividade lúdica proposta na ação extensionista. Dessa forma, podemos inferir que a estratégia lúdica "sangue que salva" contribuiu positivamente no processo de ensino-aprendizagem dos alunos do $8^{\circ}$ Ano A e B. Além de motivar e despertar o senso de responsabilidade, o trabalho em equipe e a interação professor-aluno, aluno-aluno e aluno-extensionistas. Resultados semelhantes ao observado por Silva e Antunes (2017) e Azevedo et al. (2021) ao relatarem que a atividade lúdica, por tornar as aulas mais atrativas prazerosas e dinâmicas, propiciou aos alunos autonomia, motivação e interação entre a comunidade acadêmica.

Durante a aplicação da estratégia lúdica, observou-se a participação ativa dos alunos, comparando os tipos sanguíneos e apontando de forma correta a possibilidade de ocorrer ou não a transfusão sanguínea no sentido determinado pelo jogo. As duplas debatiam entre si, e formulavam as suas respostas baseadas nos conhecimentos construídos sobre o sistema ABO e fator Rh.

Tabela 3: Análise de variância do desempenho dos alunos por turma e teste $(\alpha=0,05)$ para $n=23$ respondentes

\begin{tabular}{lccccc}
\hline & Df & Sum SQ & Mean sq & Estatística F & p-valor \\
\hline Fator Turma & 1 & 62,63 & 62,63 & 17,97 & 0,000 \\
Fator Teste & 1 & 40,2 & 40,2 & 11,53 & 0,001 \\
Resíduos & 43 & 149,89 & 3,49 & - & - \\
\hline
\end{tabular}

Fonte: Autores.

Uma vez constatado que o desempenho dos estudantes foi estatisticamente maior no pós-teste e que a turma B teve mais êxito, avaliou-se o tamanho das diferenças desse desempenho. Uma forma usual de avaliar o tamanho do efeito de diferentes grupos sobre uma variável é por meio da medida d de Cohen (1992), uma vez que os tamanhos dos dois grupos eram semelhantes e os desvios padrão similares, conforme critério de classificação proposto por Cohen (1992) para testes de diferença entre médias de grupos independentes (Espírito Santo \& Daniel, 2017; Lacruz \& Américo, 2018).

Para a comparação de média observada no pré-teste e pós-teste, foi obtido d=0,851, valor considerado grande. Esse resultado permite concluir que os estudantes foram muito mais bem sucedidos no pós-teste do que no pré-teste, quando ainda não tinham participado das atividades lúdicas. Resultado superior ao de Lacruz e Américo (2018) que observaram efeito médio entre 0,49 $<\mathrm{d}<0,8$ e de Tannenbaum e Cerasoli (2013), cuja meta-análise retornou um d =0,67. Porém, ressalta-se que a comparação pode ser limitada pelos diferentes contextos sociais, educacionais e regionais.

Na comparação da média por turma, constatou-se um efeito grande, d=1,132. Para esta variável, conclui-se, portanto, que a turma B teve desempenho muito acima do observado na turma A.

Para avaliar a variabilidade das respostas por questão, utilizou-se o coeficiente alfa de Cronbach $(\alpha)$, indicador de confiabilidade de instrumentos avaliativos com ampla utilização (Hair \& Babin, 2009). No pré-teste, foi observado $\alpha_{\text {pré }}=$ 0,559, indicativo de alta variabilidade das respostas e, portanto, baixa precisão do instrumento nesse primeiro momento. Já no pós-teste, quando os estudantes já haviam participado da estratégia lúdica, o coeficiente foi $\alpha_{\text {pós }}=0721$. Esse valor indica que o 
instrumento é aceitável para fins de avaliação dos respondentes quanto ao conteúdo estudado, sistema imunológicos, tipo sanguíneo e fator Rh (Hair \& Babin, 2009). Outros índices clássicos utilizados para avaliação de testes estão exibidos na Tabela 4 .

No que se refere ao poder discriminativo das questões, isto é, se elas separam sujeitos proficientes dos que não possuem domínio do conteúdo, verificou-se que no pré-teste as questões 1 (p_b=0,137) e 6 (p_b=0,215) não possuem poder discriminativo aceitável. Por outro lado, no pós-teste, todos os itens possuem bom poder discriminativo (Rabelo, 2013).

A respeito do nível de dificuldade clássico do teste, as proporções de erros por questão forneceram um bom indicativo (Pasquali, 2017). No pré-teste, os estudantes tiveram mais dificuldade nas questões 8 (DIF=0,957) e 10 (DIF=0,826). No pósteste, constatou-se mais dificuldades nas questões 4 e 7 (DIF=0,609).

Tabela 4: Proporções de acertos e erros por questão e medida de discriminação (correlação ponto bisserial)

\begin{tabular}{cccc|ccc}
\hline \multirow{2}{*}{ Questões } & \multicolumn{3}{c|}{ Pré-teste* } & \multicolumn{2}{c}{ Pós-teste } \\
\cline { 2 - 7 } & $\begin{array}{c}\text { Proporção de } \\
\text { Erro (DIF) }\end{array}$ & Acertos & $\begin{array}{c}\text { Correlação ponto } \\
\text { bisserial }\left(\boldsymbol{p}_{b}\right)\end{array}$ & $\begin{array}{c}\text { Proporção de } \\
\text { Erro (DIF) }\end{array}$ & $\begin{array}{c}\text { Acertos } \\
\text { Correlação ponto } \\
\text { bisserial }\left(\boldsymbol{p}_{b}\right)\end{array}$ \\
\hline 1 & 0,130 & 0,870 & 0,137 & 0,217 & 0,783 & 0,492 \\
2 & 0,348 & 0,652 & 0,630 & 0,174 & 0,826 & 0,557 \\
3 & 0,565 & 0,435 & 0,582 & 0,217 & 0,783 & 0,538 \\
4 & 0,652 & 0,348 & 0,578 & 0,609 & 0,391 & 0,496 \\
5 & 0,304 & 0,696 & 0,378 & 0,174 & 0,826 & 0,508 \\
6 & 0,609 & 0,391 & 0,215 & 0,565 & 0,435 & 0,505 \\
7 & 0,565 & 0,435 & 0,582 & 0,609 & 0,391 & 0,688 \\
9 & 0,957 & 0,044 & 0,575 & 0,478 & 0,522 & 0,459 \\
10 & 0,348 & 0,652 & 0,398 & 0,087 & 0,913 & 0,541 \\
\hline
\end{tabular}

Fonte: Autores.

Na Figura 7 observa-se o índice de dificuldade (ID) das questões, segundo Condé (2001), de acordo com o critério exposto, duas questões do pré-teste foram consideradas fáceis: Q1 e Q5. Seis questões foram classificadas de média dificuldade: Q2, Q3, Q4, Q6, Q7 e Q9, e, duas questões foram consideradas difíceis: Q8 e Q10. Sendo que a questão mais difícil foi a 8 , que apresentou apenas $4 \%$ de acerto, indicando que a maioria dos alunos não tinham conhecimento prévio do assunto da questão. Já nos estudos realizados por Duarte et al. (2020), metade das questões no pré teste, foram classificadas como de média dificuldade e metade como difíceis. Os mesmos autores destacaram que nenhuma das 10 questões foi classificada como fácil, diferente ao obtido no presente trabalho. 
Figura 7: Acertos e erros por questão em percentual: pré-teste.

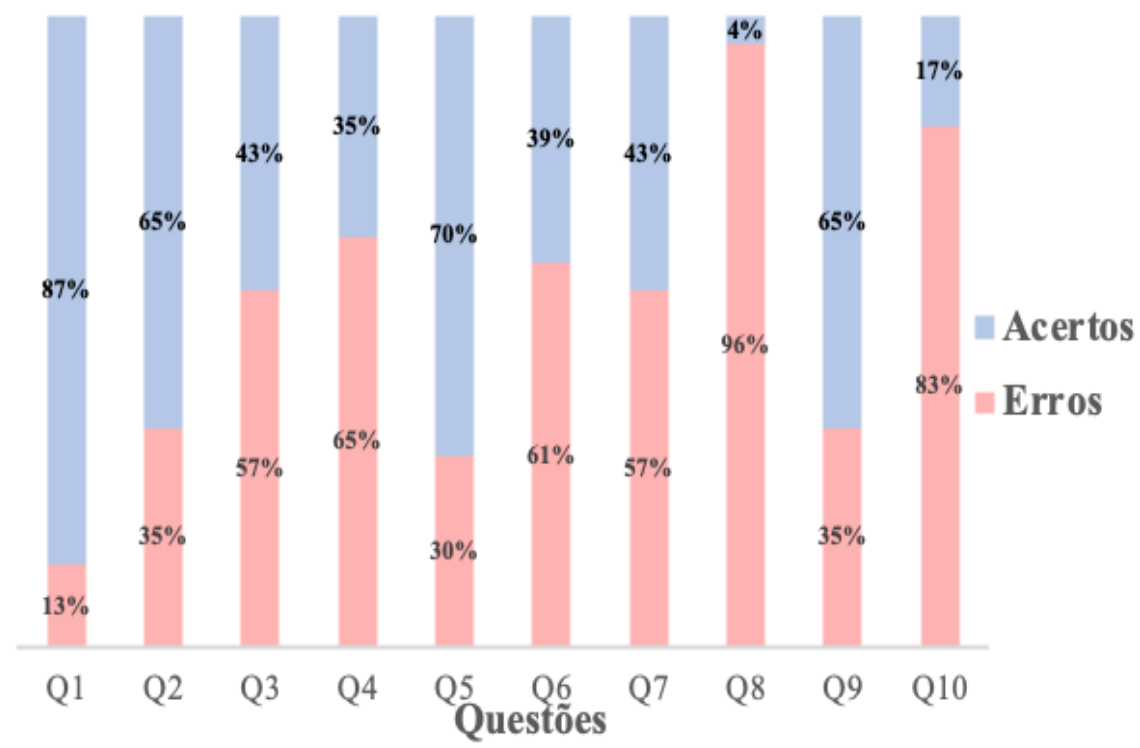

Fonte: Autores.

No pós-teste (Figura 8), houve uma diminuição do índice de dificuldade das questões, este comportamento era o esperado, uma vez que o conteúdo já havia sido ministrado na turma. Assim, no pós-teste, aumentou o número de questões fáceis de dois, no pré-teste, para cinco (Q1, Q2, Q3, Q5 e Q9) e de questões de média dificuldade, de 4 no pré-teste, para cinco: Q4, Q6, Q7, Q8 e Q10. A questão 1, no pré-teste, foi a mais fácil, com percentual de acerto de 87\%. No pós-teste a questão 9 foi a mais fácil com $91 \%$ de acerto. Ressalta-se que não houve questões difíceis no pós-teste. No entanto, mesmo com o aumento de respostas corretas no pós-teste, o percentual de erros, principalmente nas questões 4 e 7 , ainda foram altos.

Figura 8: Acertos e erros por questão em percentual: pós-teste.

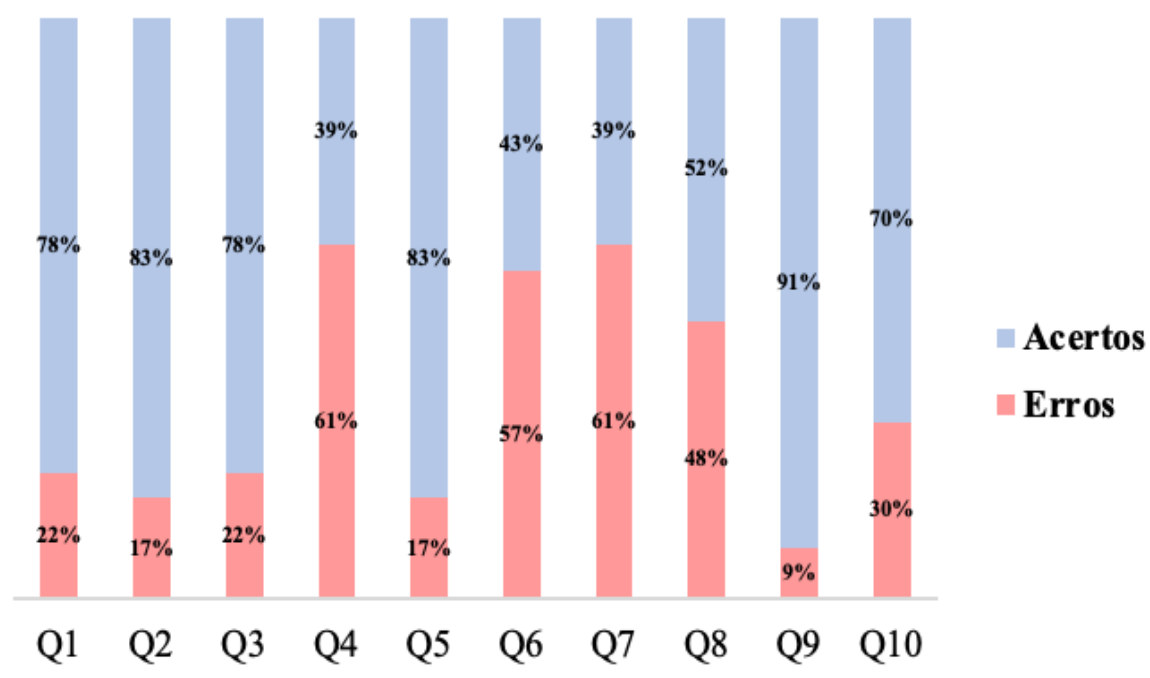

\section{Questões}

Fonte: Autores.

Para a análise da aplicação da metodologia e dos aspectos de ensino e aprendizagem, verificou-se as respostas dos alunos ao questionário aplicado. A Figura 9, mostra os resultados dos questionamentos, no qual os alunos deveriam responder 
"sim” ou "não" para as perguntas. Por meio dessas indagações, verificou-se que a maioria dos alunos aprovaram a utilização das atividades lúdicas para complementar o entendimento das aulas teóricas, essas atividades contribuíram para a assimilação do conteúdo e aprendizagem dos alunos, despertando-lhes ainda o interesse de participar de outras atividades como esta. Para Moreira, Costa, Barbosa e Bertini (2012), os jogos lúdicos além de facilitar a aprendizagem, tornam as aulas mais atrativas, divertidas e interessantes, motivando e contribuindo para o desenvolvimento de habilidades dos alunos e para a socialização da turma.

Na avaliação metodológica, solicitou-se aos alunos que avaliassem a abordagem do conteúdo pelos extensionistas, a qualidade do material de apoio das aulas, e os seus próprios conhecimentos após as aulas do projeto, como demonstrado na Figura 10. Analisando os percentuais relacionados a essa avaliação, cerca de $91 \%$ dos alunos classificaram a abordagem dos extensionistas entre 8 à 10, em relação a qualidade do material de apoio $87 \%$ dos alunos classificaram o material entre 8 à 10 , e na avaliação dos seus próprios conhecimentos após as atividades do projeto $82 \%$ dos alunos classificaram seus conhecimentos entre 7 à 10, esses resultados demonstram que, para a maioria dos alunos, a abordagem dos extensionistas e o material de apoio das aulas, contribuíram para a sua aprendizagem. De acordo com Mesquita et al. (2017), o uso de recursos educativos favorece o processo de interação entre os pares, fazendo-se um elemento influente na aprendizagem, dessa forma, contribuindo para o público entender as temáticas e para o estabelecimento do diálogo e da troca de conhecimentos.

Figura 9: Avaliação da metodologia por parte dos estudantes que participaram dos dois testes

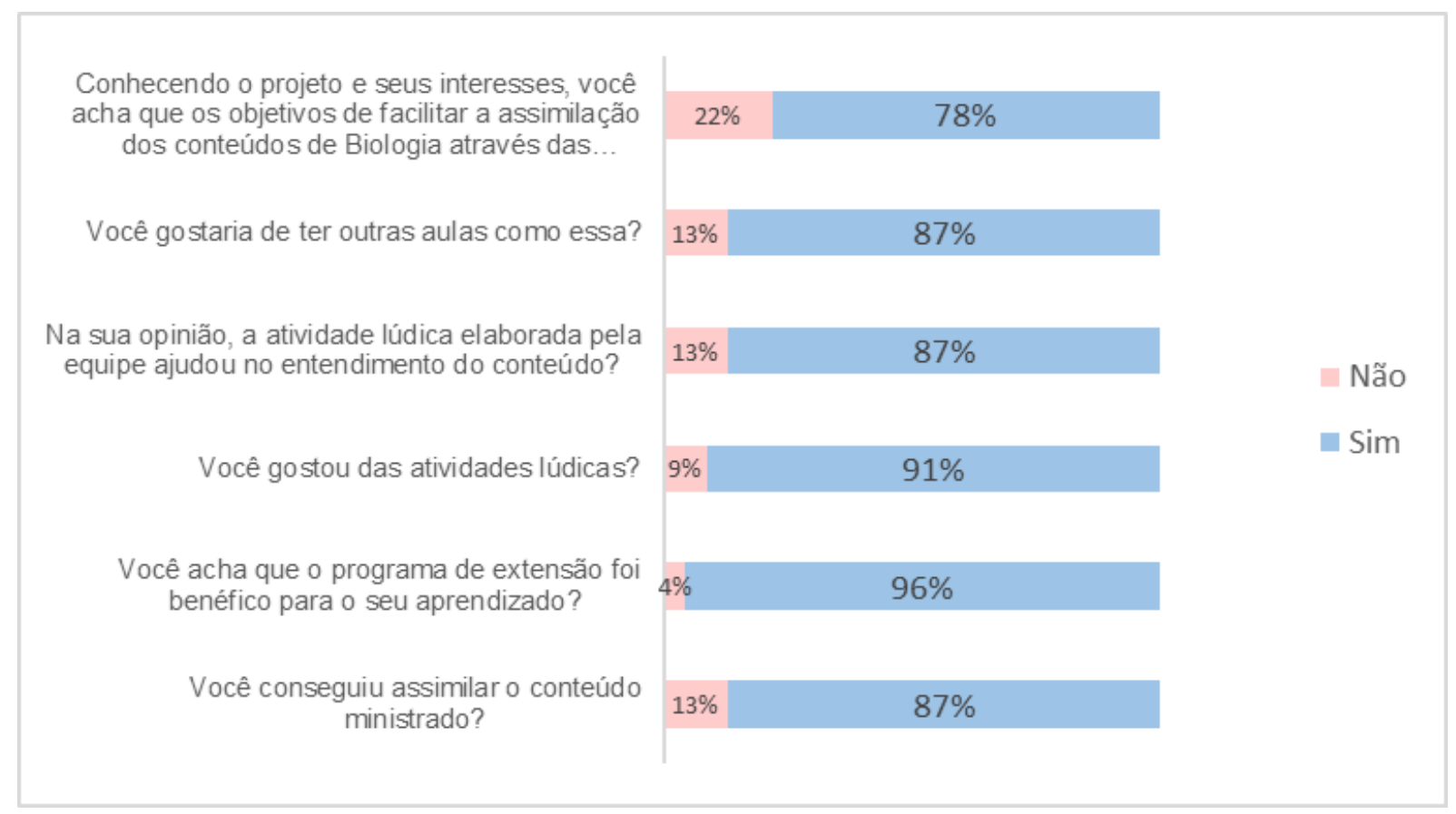

Fonte: Autores. 
Figura 10: Avaliação da metodologia por parte dos estudantes que realizaram participaram dos dois testes

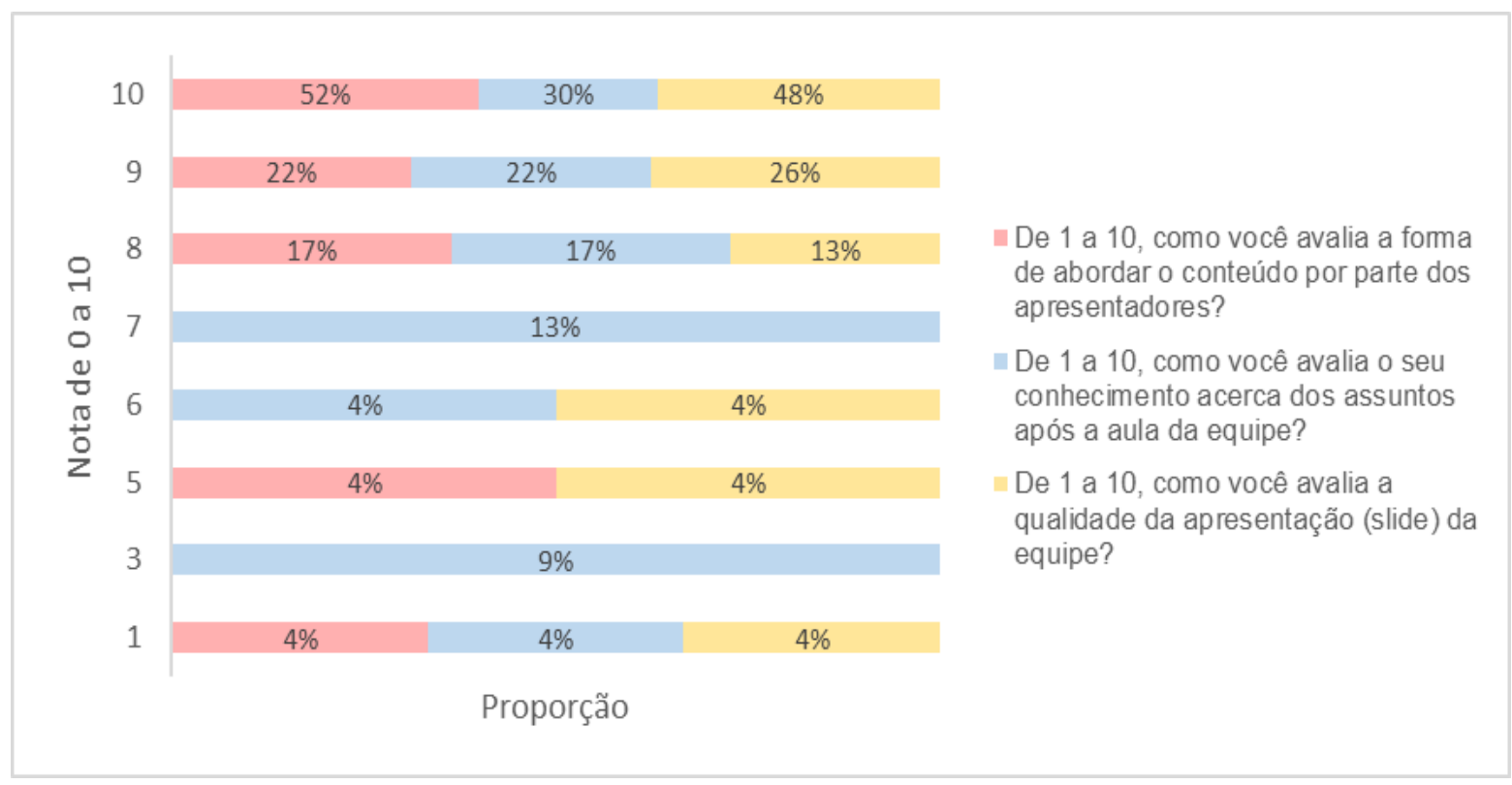

Fonte: Autores.

\section{Conclusão}

Esta pesquisa partiu do entendimento de que o êxito no processo de ensino e aprendizagem é alcançado quando se oportuniza as condições adequadas para o estudante explorar e (re) construir os conhecimentos. Em uma ação contrária, isto é, com a adoção de uma linha de ensino enrijecida e única, a aprendizagem pode ser prejudicada.

Sob essa perspectiva, o estudo foi pautado na natureza lúdica, como estratégia principal para o exercício e o desenvolvimento de habilidades intelectuais. Assim, em atenção ao objetivo geral, a pesquisa consistiu em aplicar a ludicidade como uma alternativa didática no ensino das ciências biológicas e, consequentemente, identificar as possíveis contribuições ao processo educacional.

Os resultados ratificaram que a educação é complexa à medida que se permite refletir e acenar para a resistência. Logo, os dados tornaram perceptível que as diferenças entre os desempenhos das turmas consultadas refletem diversos fatores sociais (externos e internos), como, a socialização, o nível de escolaridade e o acesso aos meios de informação. Outrossim, ficou claro que ao possibilitar o espaço para que o estudante utilize os seus conhecimentos prévios e os relacione ao novo conteúdo, a educação se torna mais significativa.

É possível a reflexão de que a visão da importância da ludicidade pode ter alcançado ainda o(s) docentes(s) da escola, no desenvolvimento de seus planejamentos e na disponibilização de subsídios à prática pedagógica. Em geral, é imprescindível a construção da via de mão dupla na dinâmica educacional: o professor mediar e estimular a aprendizagem, para que o estudante interaja e resolva as situações-problema.

Destarte, em função de o estudo ter constatado que as metodologias lúdicas instigam a construção do conhecimento pelos estudantes, e a ludicidade não se reduz aos materiais pedagógicos - mas, estabelecida também através da postura do docente para rompimento da distância entre o real e abstrato, levando o discente a sentir prazer pelo universo científico, sugere-se um estudo sobre a relação entre professor, estudante e conhecimento, isto é, se a afetividade e as relações interpessoais em associação com as metodologias ativas e lúdicas favorecem o alcance de aprendizagem significativa. Um estudo dessa natureza se reforça pelo impacto da pandemia na educação, cujas práticas de ensino presenciais foram substituídas pelo universo remoto. 


\title{
Agradecimentos
}

\begin{abstract}
À Pró-Reitoria da Cultura, Comunidade e Extensão - Procce/Ufopa, pelo suporte na realização da pesquisa.
À Escola Municipal de Ensino Fundamental Paulo Rodrigues dos Santos, pelo apoio à realização do trabalho.
\end{abstract}

\section{Referências}

Azevedo, M. M. R., Vieira, D. D. S. S., Hager, A. X., Vieira, J. C., Vieira, A. C., Sousa, E. T. F., Vieira, L. A., \& Pereira, R. J. B. (2021). Kahoot como estratégia lúdica no ensino aprendizagem de Biologia Celular. Research, Society and Development, 10(12), e159101219049. https://doi.org/10.33448/rsdv10i12.19049

Cohen, J. (1992). Power primer. Psychological Bulletin, 112 (1), 155-159. https://doi.org/10.1037/0033-2909.112.1.155

Condé, F. N. (2001) Análise empírica de itens (Relatório técnico). Instituto Nacional de Estudos e Pesquisas Educacionais Anísio Teixeira. Brasília, DF.

Duarte, C. T. de S.; Dorabiato, M. D.; Azevedo, M. M. R.; Hager, A. X. Estratégia lúdica no processo de ensino-aprendizagem da biologia no ensino superior (Cap. 5). In: Siebert, P. R.; Sousa, G. dos S. de.; Siebert, T. H. R. (ORGS.), Pesquisas e reflexões sobre o ensino de ciências e matemática na região do Baixo Amazonas (pp. 83-100). Belém-PA: RFB. https://doi.org/10.46898/rfb.9786558891147.5

Espírito Santo, H., \& Daniel, F. (2017). Calcular e apresentar tamanhos do efeito em trabalhos científicos (1): as limitações do $\mathrm{P}<0$, 05 na análise de diferenças de médias de dois grupos (Calculating and Reporting Effect Sizes on Scientific Papers (1): P< 0.05 Limitations in the Analysis of Mean Differences of Two Groups). Revista Portuguesa de Investigação Comportamental e Social, 1(1), 3-16. https://doi.org/10.7342/ismt.rpics.2015.1.1.14

Feijó, N., \& Delizoicov, N. C. (2016). Professores da educação básica: Conhecimento prévio e problematização. Retratos da Escola, 10(19), 597-610. https://doi.org/10.22420/rde.v10i19.643

Fenner, A. L., \& Corbari, A. T. (2005). O conhecimento prévio do aluno: um alicerce para a aprendizagem significativa de língua estrangeira. Tempo da Ciência, 12(24), 09-15. https://doi.org/10.48075/rtc.v12i24.443

Freitas Filho, J. R. de, Freitas, J. C. R. de, Silva, L. P. da, \& de Melo, R. C. L. (2012). Brincoquímica: uma ferramenta lúdico-pedagógica para o ensino de Química Orgânica. XVI ENEQ/X EDUQUI-ISSN: 2179-5355. Pernambuco (RE).

Gil, A. C. Métodos e técnicas de pesquisa social. 6. ed. São Paulo: Atlas, 2008.

Hair, J. F., Black, W. C., Babin, B. J., Anderson, R. E., \& Tatham, R. L. (2009). Análise multivariada de dados (6a ed., p. 688). Porto Alegre: Bookman.

Lacruz, A. J., \& Américo, B. L. (2018). Influência do debriefing no aprendizado em jogos de empresas: um delineamento experimental. BBR. Brazilian Business Review, 15, 192-208. http://dx.doi.org/10.15728/bbr.2018.15.2.6

Marques, K. C. D. (2018). Modelos didáticos comestíveis como uma técnica de ensino e aprendizagem de biologia celular. Tear: Revista de Educação, Ciência e Tecnologia, 7(2), a3177. https://doi.org/10.35819/tear.v7.n2.a3177.

Moreira, F. B. D. F., Costa, M. D. O., Barbosa, E. M., \& Bertini, L. M. (2012). Bingo Químico: Uma atividade lúdica envolvendo fórmulas e nomenclaturas dos compostos. HOLOS, vol. 6 .

Paim, M. C., Goldschmidt, A. I., \& Loreto, E. L. S. (2021). Concepções prévias de alunos do $9^{\circ}$ ano do Ensino Fundamental sobre o processo de cicatrização e sua relação com a Biologia Celular. Research, Society and Development, 10(8), e10610817000. https://doi.org/10.33448/rsd-v10i8.17000

Pasquali, L. (2017). Psicometria: teoria dos testes na psicologia e na educação. Petrópolis: RJ, Vozes.

Pereira, R. J. B., Azevedo, M. M. R., Sousa, E. T. F., \& Hager, A. X. (2020). Método tradicional e estratégias lúdicas no ensino de Biologia para alunos de escola rural do município de Santarém-PA. Experiências em Ensino de Ciências, 15(02), 106-123.

Pliessnig, A. F. (2013). Jogo Sangue que Salva. Núcleo Regional de Educação de Telêmaco Borba. http://www.biologia.seed. pr.gov.br/modules/conteudo/conteudo.php?conteudo $=1326$

Rabelo, M. (2013). Avaliação educacional: fundamentos, metodologia e aplicações no contexto brasileiro. Rio de janeiro: SBM, 29, 30-31.

Ribeiro, A. F. S. (2019). Jogo educativo digital como apoio no processo de construção do conhecimento: possibilidades e potencialidades ao ensino de Sistemas de Telecomunicações. Dissertação de mestrado, Instituto Federal Goiano, Morrinhos, GO, Brasil.

Silva, M. R. da, \& Antunes, A. M. (2017). Jogos como tecnologias educacionais para o ensino de genética: a aprendizagem por meio do lúdico. Revista Eletrônica Ludus Scientiae, 1(1). https://doi.org/10.30691/relus.v1i1.660

Sousa, E. T. F., Azevedo, M. M. R., Hager, A. X., Brito, A. E. de O., Silva, L. P. da., Pereira, R. J. B., Góes, A. K., Bressan, C. R., Lima, J. S. de, \& Pereira, K. P. (2021). A estratégia lúdica como mediadora da educação em genética em uma escola pública. Research, Society and Development, 10(13), e261101320939. https://doi.org/10.33448/rsd-v10i13.20939

Sousa, N. (2017). Planeamento experimental usando ANOVA de 1 e 2 fatores com R: uma breve abordagem prática. Universidade Aberta. Lisboa: 01-26.

Tannenbaum, S. I., \& Cerasoli, C. P. (2013). Do team and individual debriefs enhance performance? A meta-analysis. Human factors, 55(1), 231-245. https://doi.org/10.1177/0018720812448394

Torres, J. P., \& Mendes, E. G. (2019). Atitudes Sociais e Formação Inicial de Professores para a Educação Especial. Revista Brasileira de Educação Especial, 25(4), 765-780. https://doi.org/10.1590/s1413-65382519000400014 